\title{
ANALYSIS OF AN INTELLIGENT TEMPERATURE TRANSMITTER FOR PROCESS CONTROL
}

\author{
0. V. Okoro ${ }^{1},{ }^{*}$ C. C Osuagwu ${ }^{2}$ and I. O. Okeke 3

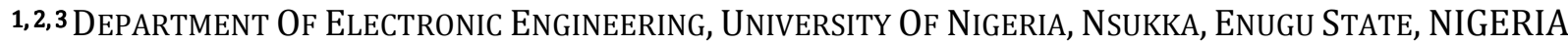 \\ Email addresses:1 okiteval@gmail.com,2 ccosuagwu@hotmail.co.uk,3ijeoma.okeke@unn.edu.ng
}

\begin{abstract}
In recent times, transmitters that incorporate microprocessor to perform various intelligent functions have been developed by various manufacturers. This paper presents an overview of the evolution in transmitter technology while highlighting key factors which have influenced the evolution. It also identifies low power microprocessor and analog to digital converters working with the basic sensor circuit as the key propellants in the advancement of transmitter technology. Despite several sensors available in the process control industry, the authors focus on temperature sensors and analyze a typical Rosemount Intelligent Temperature Transmitter (RITT) with a view to identifying and comparing how experimental results vary from established parameters in the datasheet. Simulation results show that the resistance of the RITT's Resistance Temperature Detector (RTD) varies directly with applied temperature. Percentage error shows acceptable points at $-0.04 \%, 0.04 \%$ and $-0.1 \%$. For higher percentage error readings, it is necessary to connect a resistor of value between $250 \Omega$ and $1100 \Omega$ between the current loop and the transmitter. The future of transmitter technology is however the wireless sensor node (WSN) incorporating the Sensirion SHT11 temperature sensor.
\end{abstract}

Keywords:-intelligent transmitter, wireless sensor node, rosemount intelligent temperature $\quad$ transmitter, field bus, process industry, telos $B$.

\section{INTRODUCTION}

Transmitter in process control refers to a device that translates the signal produced by a primary sensing element like a temperature sensor into a standardized instrumentation signal such as 3-15 PSI air pressure, 420mA DC electric current or a Fieldbus digital signal. These signals are then conveyed to an indicating device, a controlling device or both [1]. The primary sensing element in this context is a device such as platinum RTD, negative temperature coefficient thermistor or thermocouple device. These devices directly sense the process variable and translate the sensed quantity into an analog representation (voltage, current, resistance, mechanical force or motion). Over the years, several types of transmitters have been employed to sense, control and influence process variables like temperature, pressure, flow, level and even certain types of gases. With technological advancement and the increased need for cost-effective and reliable equipment deployment, transmitters have had to evolve over the years in order to meet these constantly changing user demands.

The evolution in the design of transmitters has been influenced by the requirements of users for improved performance, reduced cost of ownership as well as developments in adjacent technologies such as Computer Aided Design (CAD), microelectronics, material science and communication technologies [2]. Low power microprocessors and analog to digital converters functioning in conjunction with the basic sensor circuit have caused the most significant advancement in transmitter design [2]. This is especially true because of increased performance experienced in the use of low power microprocessors and analog to digital converters with transmitters. This enables non-linear characteristics to be corrected and also enables a secondary sensor to be included in transmitters, ultimately enabling secondary effects on the primary sensor to be compensated. This has enabled the evolution from an era in which only the measurement signal is transferred, to one in which the 
microprocessor not only implements its processorbased functions but also manages a communication facility, thus allowing data specific to the transmitter such as type, serial number and transmitter address to be stored at the transmitter and called up when required. Amore recent development in transmitter evolution involves multiplexing the transmitter's output onto a network or Fieldbus using Object Linking and Embedding (OLE) for Process Control (OPC). OLE is an international standard and enables devices from different manufacturers to be interoperable.

In this paper, Rosemount 3144P intelligent temperature transmitter's Platinum PT 100 RTD, employed in process control is analyzed with a view to comparing experimental findings with manufacturer's details in datasheet. The aim is to understand and compare how characteristics like temperature, accuracy and temperature coefficient differ in real life applications from expected variables in datasheet.

The future of transmitters however is WSN. These devices are generally equipped with sensing, data processing and communication capabilities [3]. The sensing circuitry measures ambient conditions relating to the environment surrounding the sensor, after which sensed parameters are transformed into electric signal. Processing such signals reveal properties about objects located and/or events happening in the vicinity of the sensor.

The rest of the paper is organized as follows: section 2 gives an overview of sensor technologies. Section 3 discusses intelligent temperature transmitters, communication protocols and sensors. Section 4 presents the experimental design while section 5 discusses the results. Section 6 concludes the paper.

\section{OVERVIEW OF SENSOR TECHNOLOGIES}

The range of microprocessor based measuring systems currently in use cover a wide variety of applications like military, environmental, health, home and industrial applications. Those that are intelligent have however been specifically deployed for use in industrial applications, particularly the process control industry [2] with temperature, pressure and differential pressure transmitters taking the lead in the development of these intelligent sensors.

In recent times, transmitters have the capability to communicate with a remote instrument over the same network that carries measurement signal. With remote communication as well as two-way communication over the same circuit now possible, the need for a standard that enables these enhanced capabilities is highly desirable. The 4-20mA transmission standard, for example has greatly developed process instrumentation during the past three decades [2]. Some benefits of the $4-20 \mathrm{~mA}$ transmission standard thus include [2]:

1. Measured signal is unaffected by loop current.

2. Enables inter-manufacturer transmitter compatibility

3. Facilitates the inclusion of alarms without affecting the accuracy of measurement

However, a major limitation to the $4-20 \mathrm{~mA}$ transmission standard is that only the measured signal and not transmitter information can be transmitted [2]. This limitation, in addition to the requirement for twoway communication led to the introduction of Fieldbus. Fieldbus enables two-way communication which reduces cost of wiring, commissioning and maintenance times and increases versatility and functionality [2]. Examples of fieldbus interfaces include Highway Addressable Remote Transducer (HART) field communication protocol, FOUNDATION Fieldbus, process field bus-decentralized peripherals (PROFIBUS-DP), control area network bus (CANbus), control network (Contro INET), device network (Device NET)[1].

The Global Agenda Council on Emerging Technologies has however identified wireless power as one of the top 10 emerging technologies for 2012 [4]. These cheap, smart devices with multiple onboard sensors and networked through wireless links and the internet can now be deployed in large numbers to provide solutions in the same way as the traditional intelligent transmitters. A lot of research is still ongoing in order to combat the limitations of wireless sensor network which include constrains in energy supply, storage capacity and bandwidth not to mention the need for the deployment of large number of these sensor nodes in an area of interest [5].

\section{INTELLIGENT TRANSMITTER}

In the view of Boyles [2], intelligent transmitters are transmitters that incorporate a microprocessor that derives the primary measurement signal. These transmitters are also capable of storing information about the transmitter, its application data and location, in addition to managing two-way communication between the transmitter and a communication interface such as a Fieldbus.

Transmitters are often required to be deployed in particular locations in order to carry out specific measurements, especially those requiring a wide range 
of measurement. Intelligent transmitters now permit the same instrument to be used for large or small measurement ranges which is a limitation for ordinary transmitters. An intelligent transmitter can provide the same analog output onto a digital communication network. This enables one transmitter to be configured to deal with a range of applications like its primary sensing and the display of device parameters, resulting in fewer transmitters being deployed in the field. Other advantages of intelligent transmitters include: improved commissioning time since the transmitters can be addressed from the control room or marshalling cabinets; increased reliability with in-built diagnostic routine and reduced need for repairs and maintenance because the communication facility facilitates better maintenance record keeping.

\subsection{Microprocessor-based Intelligent Temperature Transmitter}

Several companies now manufacture intelligent temperature transmitters. The authors have however chosen the Rosemount 3144P temperature transmitter as a case study, comparing its Platinum PT 100 RTD sensing characteristics with information obtained from datasheet. Rosemount Model 3144Pintelligent temperature transmitter is amicro processor based instrument that accepts input from a wide variety of sensors and transmits captured temperature data to a control system or transmitter interface. This model transmits data via a HART interface as indicated in the datasheet [6].

\subsection{Fieldbus Communication Protocol}

Most microprocessor based intelligent transmitters operate in the conventional $4-20 \mathrm{~mA}$ measurement circuit. This is gradually changing as mentioned in section 2 due to the numerous advantages to be gained from multiplexing signals from sensors, transmitters and controllers. Any digital network designed to interconnect field-located instruments can be termed a Fieldbus. The use of Fieldbus with transmitters thus enables full advantage to be taken of the enhanced performance of sensors, enabling a wide range of information to be extracted from the transmitter on request as well as adjustments to be made to the transmitter's operating mode. An example of such an adjustment on the transmitter's operating mode is adjustment of span and zero to permit the full range of the analog output signal (0-20mA or $4-20 \mathrm{~mA})$. Two types of fieldbus discussed in this paper are:
1. HART Protocol: HART is the most used communication protocol in automation industrial applications requiring real-time support with a device count around 20 million [7]. In this protocol, sine wave of digital signals which make up $1.2 \mathrm{KHz}$ and $2.2 \mathrm{KHz}$, representing bits 1 and 0 respectively are superimposed using frequency shift keying modulation on top of a 4-20mA analog current loop between different components, thus resulting in simultaneous analog and digital communication[1] [8]. Key features of HART thus include: the possibility of rearranging field instrument remotely using the HART communicator and the ability to programme field instruments with an identification data [1].

2. FOUNDATION Fieldbus (FF): This is an all digital and serial two-way communication system interconnecting field equipment such as sensors, actuators and controllers and having current from a constant current supply, digitally modulated. FF is based on IEC 61158-2 physical layer standard and is capable of providing both power and communication on the same pair of wires [2]. Two types of FF protocols exist, namely FF $\mathrm{H} 1$ and FF High Speed Ethernet (HSE) [9]. A striking feature that $\mathrm{FF}$ has is that it is not only a communication protocol but also a programming language. Its graphical programming language can thus be used for building control strategies and employs an extension library of function blocks for input, output control and arithmetic calculations [2]. Other advantages of Foundation Fieldbus include increased speed, unlimited addresses and standardization; although its limitation is that a failure to the bus can shut down the entire process.

\subsection{Sensors}

Sensors are devices that convert a physical phenomenon into an electrical signal, thus translating aspects of physical reality into representations that are understandable and can be processed by computers [10].Most sensors do not directly produce voltage but rather act like passive devices, an example being a resistor, whose values change in response to external stimuli. In order to produce voltages suitable for input into microprocessor and their analog-to-digital converters, the resistor must thus be "biased" (made to behave in a particular manner) and the output signal amplified.

Categorizing sensors may either be based on their principle of operation or by the function that they 
perform, although the more logical way to classify sensors as opined by Ripka and Tipek [11] is with regard to the physical property they measure. This result in the following categorization: mechanical, thermal, electrical, magnetic, radiant, chemical and biochemical.

\subsection{Temperature Sensors}

Temperature is one of the most widely sensed of all parameters in the world today alongside pressure [2]. Temperature sensors detect a change in a physical parameter (resistance or output voltage) that corresponds to a temperature change. A temperature sensor can thus be contact type: RTDs, thermocouples or thermistors; or non contact type: infrared and acoustic [8]. Contact type temperature sensors are briefly discussed and have the following characteristics [8] [10-11]:

1. Electronic Temperature Sensor. These are thermocouples and are characterized by the seedback effect, i.e., when a pair of dissimilar metals are joined at one end, and there is a temperature difference between the joined end and the open end, thermal electromagnetic force is generated, creating a flow of current through the wires that is proportional to the temperature difference.

2. Resistive Temperature Sensors: The resistance of these sensors changes with the temperature. A very important example is the thermistor, which is a type of resistor with resistance varying according to its temperature.

3. Resistive Temperature Detectors (RTDs): They are made from pure metal (copper, nickel or platinum) and are useful over large temperature range. They require measuring current to generate a useful signal. A typical RTD called platinum PT 100 is shown in Fig 1.

\subsection{Wireless Sensor Node}

WSN are cheap, smart devices that have data processing and communication capabilities and perform data gathering, processing, and coordination and management functions in a collaborative way
[5][13]. WSNs, are a form of intelligent transmitter with the added advantage that they are self-configured and infrastructure less and also capable of monitoring and capturing physical/environmental parameters such as temperature, sound, vibration, pressure, motion or pollutants. Captured parameters are then cooperatively passed through the network via radio signal to a main location or sink where they can be observed and analyzed. Some commercially available WSNs in today's market are illustrated in Fig 2 and their properties listed in Table 1. WSN has key advantages of being cheap, efficiently utilizing available resources (power, storage and communication); have low cost of deployment, troubleshooting, maintenance and repairs; is flexible, adaptive (topology control) and extremely scalable.
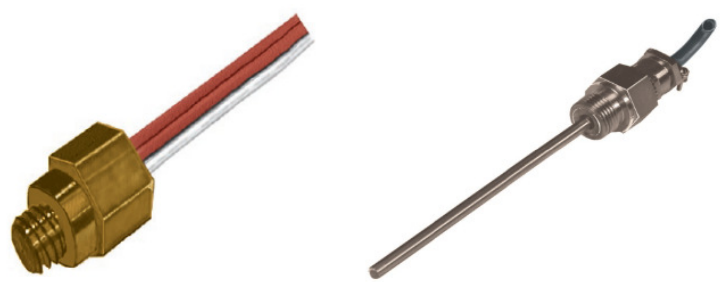

Fig 1 Platinum PT 100 RTD [12]

\subsubsection{CM5000 Sensor Node}

The CM5000 is a Telos B sensor node and is IEEE 802.15.4 compliant in addition to having the following characteristics [14]:

1. IEEE 802.15.4 WSN platform

2. TI MSP430 processor

3. CC2420 radio frequency transceiver

4. TinyOS and ContikiOS compatible operating system

5. Temperature, humidity and light sensors

6. Universal serial bus (USB) interface.

Particularly interesting with the TelosB node is the fact that in addition to its possibility of use in environmental monitoring, it has relevant application for use by developers in research [14]. The temperature sensor employed by CM5000is the Sensirion SHT11 temperature and humidity sensor and is suitable for low power applications [14].

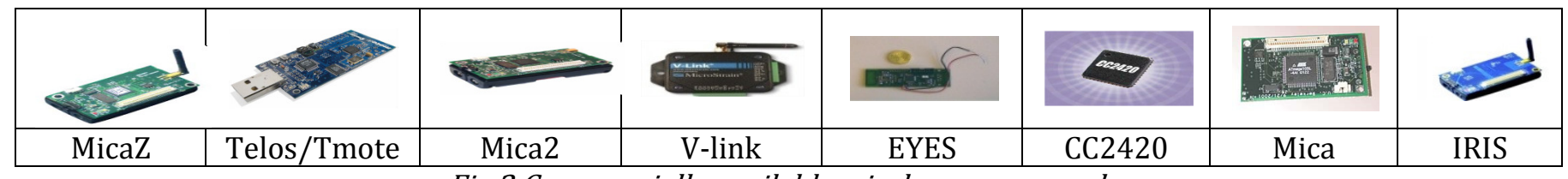

Fig 2 Commercially available wireless sensor nodes 
Table 1 Sensor node hardware

\begin{tabular}{ccccccc}
\hline S/n & Sensor node type & $\begin{array}{c}\text { CPU speed } \\
(\mathrm{MHz})\end{array}$ & Program Memory (KB) & RAM (KB) & $\begin{array}{c}\text { Radio Frequency } \\
(\mathrm{MHz})\end{array}$ & $\begin{array}{c}\text { Transmission } \\
\text { Rate (Kbps) }\end{array}$ \\
\hline 1. & Mica & 6 & 128 & 4 & 868 & $10 / 40$ \\
2. & Mica2 & 16 & 128 & 4 & $433 / 868 / 916$ & 38.4 Kbaud \\
3. & MicaZ & 16 & 128 & 4 & 2400 & 250 \\
4. & TelosB/Tmote & 16 & 48 & 10 & 2400 & 250 \\
$\mathbf{5}$ & IRIS & 16 & 128 & 8 & 2400 & 250 \\
6. & V-Llink & N/A & N/A & N/A & 2400 & 250 \\
7. & EyesIFX & 8 & 60 & 2 & 868 & 115 \\
8. & CC2420 & 16 & 48 & 10 & 2400 & 250 \\
\hline \multicolumn{7}{r}{}
\end{tabular}

These sensor nodes are also typically surface mounted, integrating sensor elements in addition to signal processing [15]. Table 2 shows the characteristics of Sensirion SHT11 temperature sensor, while fig 3 shows a typical Sensirion SHT11 chip.

Table 2 Sensirion temperature sensor characteristics

\begin{tabular}{|c|c|c|c|c|c|}
\hline Parameter & Condition & Minimum & Typical & Maximum & Unit \\
\hline Resolution & & 0.04 & 0.01 & 0.01 & ${ }^{\circ} \mathrm{C}$ \\
\hline \multirow{2}{*}{ Accuracy } & Typical & & \multirow{2}{*}{ \pm 0.4} & & \multirow{2}{*}{${ }^{\circ} \mathrm{C}$} \\
\hline & Maximum & & & & \\
\hline Repeatability & & & \pm 0.1 & & ${ }^{\circ} \mathrm{C}$ \\
\hline $\begin{array}{l}\text { Operating } \\
\text { range }\end{array}$ & & -40 & & 123.8 & ${ }^{\circ} \mathrm{C}$ \\
\hline $\begin{array}{l}\text { Source } \\
\text { Voltage }\end{array}$ & & 2.4 & 3.3 & 5.5 & VDC \\
\hline \multirow{3}{*}{$\begin{array}{c}\text { Power } \\
\text { consumption }\end{array}$} & Sleep & & 2 & & $\mu w$ \\
\hline & Measuring & & 3 & & $\mu w$ \\
\hline & Average & & 90 & & $\mu W$ \\
\hline
\end{tabular}

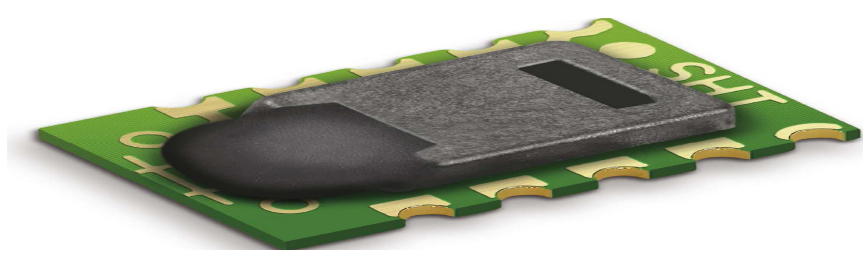

Fig 3: Sensirion SHT11 temperature sensor chip [15]

\section{EXPERIMENTAL DESIGN}

Electrical temperature sensors, such as thermocouples (T/Cs), RTDs and thermistors, produce low-level signals [8]. Thermocouples produce voltage, while RTDs and thermistors produce resistances which are proportional to temperature [8]. The 3144P transmitter converts low-level signals to HART signal and then transmits the signals to the control system via two power/signal wires. The sensing element in this case is platinum PT100 RTD. Fig 4 shows a typical wiring connection for Rosemount 3144P intelligent temperature transmitter as well as the 4-wire RTD and ohms connection adopted in the experiment. A 24VDC power supply generated by a universal Instrument bench was used to power the transmitter with connections to the positive and negative terminals as shown in fig 4. An Emerson 475 Field Communicator was then connected to the sensor terminals in parallel with a Fluke 1587 insulation multimeter to double check the current reading on both the transmitter and Field communicator displays. Figure 5 shows a typical HART communication connection with the current loop having between $250 \Omega$ and $1100 \Omega$ resistance which is essential for preventing errors due to sensor lead resistance and electrical noise.

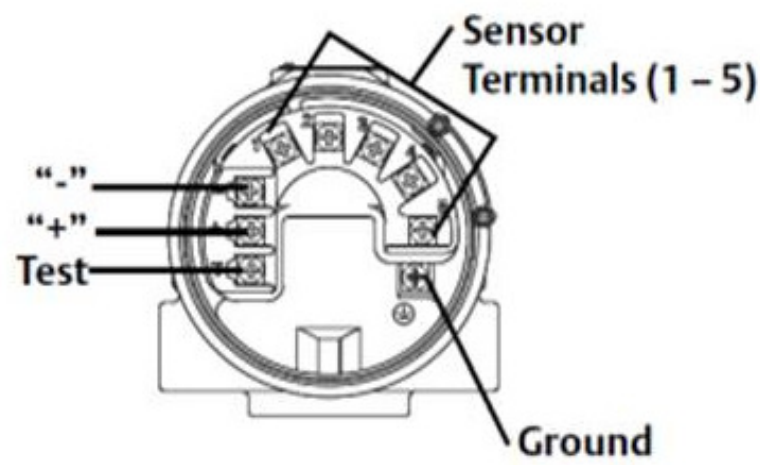

Fig 4: Wiring connections for Rosemount $3144 P$ intelligent temperature transmitter [6]

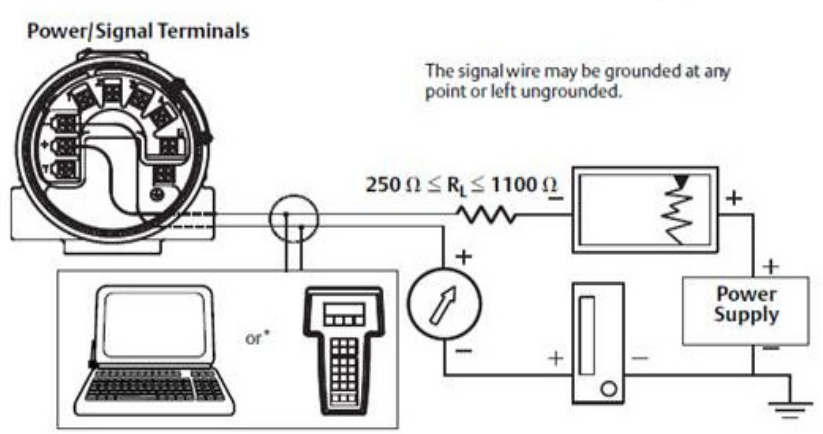

Fig 5: Connection of field communicator to a transmitter loop (HART/4-20 mA)[6] 
In the experiment, the transmitter for the RTD sensor test was identified to be factory calibrated from $0^{\circ} \mathrm{C}$ to $50^{\circ} \mathrm{C}$ as stated in the transmitter data sheet. The authors used an AMETEK JOFRA Instruments temperature calibrator (Hot Bath) to simulate heat introduction and removal. The transmitter's platinum RTD was connected to the temperature bath as seen in the experimental setup in fig 6. A stop clock was used to monitor the rise in temperature at two minutes intervals starting at $12.5^{\circ} \mathrm{C}$. After the transmitter had attained the maximum temperature of $50^{\circ} \mathrm{C}$ and the Hot Bath switched off, the fall temperatures were then taken, still using 2 minutes interval until the temperature dropped to $12.5^{\circ} \mathrm{C}$.

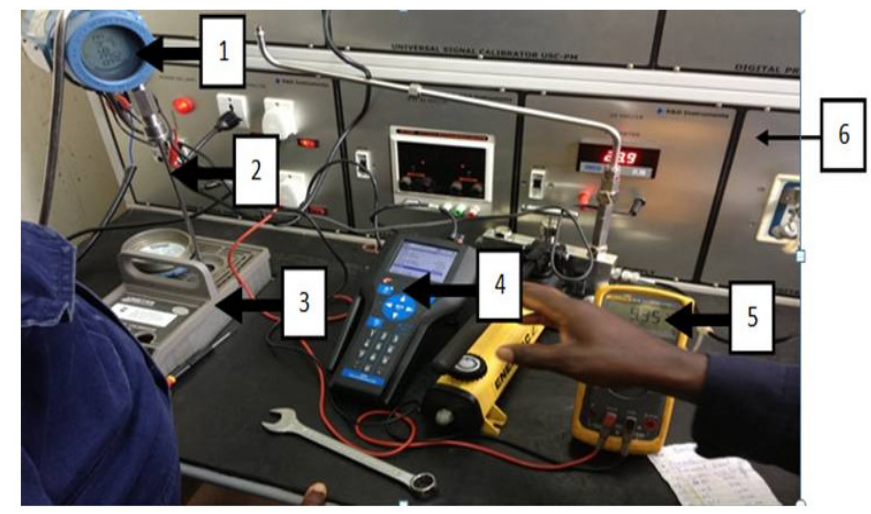

Legend: 1-Rosemount intelligent temperature transmitter, 2-Platinum PT 100 RTD, 3-

Temperature bath, 4-HART communicator, 5-Fluke multimeter, 6-Universal instrument bench

Fig 6: Experimental setup for the simulation

\section{RESULTS AND DISCUSSION OF RESULTS}

Table 3 shows the rise and fall readings obtained when the RTD of the Rosemount 3144P intelligent temperature transmitter was simulated using a temperature bath. The simulation result shown in fig 7 shows the plots for rise and fall temperature versus time. It is observed that deviations between the reading of the reference temperature (Hot Bath) and that of the transmitter exist. This shows that the RTD did not attain the same temperature as the hot bath (oven) at certain points. This error margin is however expected, though this value should be within the acceptable tolerance level (accuracy) as specified by the manufacturer, in this case $\pm 0.1 \%$ [6].

Note: the actual time for the experiment is 38 minutes for the rise and 38 minutes for the fall.

From fig 7, it can also be seen that from 0 to 30 minutes, there is a rise in temperature of both temperature bath and transmitter. Then, between 30 minutes and 40 minutes both temperatures stabilize. Finally, there is a temperature drop in both the hot bath and transmitter as heat is removed from the system.

Readings obtained from the experiment were thus used to calculate the percentage error of the transmitter as follows:

Percentage Error $=($ Error $/$ Full Scale $) \times 100 \% \ldots$ (1)

Where,

Error $=$ the Reference temperature - Transmitter temperature

Full scale is the Transmitter calibrated maximum range $\left(50^{\circ} \mathrm{C}\right)$, Applying the above equation, it is observed from Table 4 that in the rise experiment, two points meet the manufacturer's tolerance range at $0.04 \%$ and $-0.04 \%$ respectively. Percentage error for each temperature reading obtained is presented in Table 4 . Percentage error was found to be within acceptable margins at two points at $25^{\circ} \mathrm{C}$ in the rise temperature, i.e., $0.04 \%$ and $-0.04 \%$ and at one point at $25^{\circ} \mathrm{C}$ for the fall temperature, i.e., $-0.1 \%$.

Also, applying ohms law, we can deduce an equation for obtaining the resistance of the transmitter's temperature sensor (RTD) at every temperature interval.

$$
\mathrm{V}=\mathrm{IR}
$$

Where,

$\mathrm{V}$ is the Applied DC voltage which in this case is 24VDC, I is the measured current from the multimeter and transmitter display and $\mathrm{R}$ is the Resistance of temperature sensing device, in this case platinum RTD Employing the above formula, the resistance of the transmitter RTD was obtained for all simulated temperature values as shown in Table 5 .

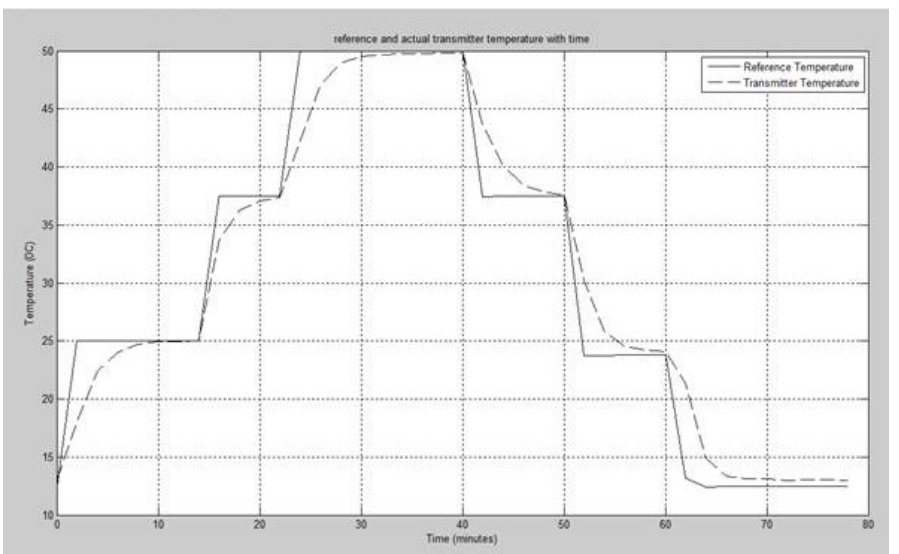

Fig 7: Simulation result plots of Function check on Rosemount 3144P RTD 
Table 3: Experimental results of a Rosemount 3144P intelligent temperature transmitter

\begin{tabular}{|c|c|c|c|c|c|c|c|c|}
\hline \multicolumn{4}{|c|}{ RISE } & \multicolumn{4}{|c|}{ FALL } & \multirow[b]{2}{*}{$\begin{array}{c}\text { Time (minutes) } \\
\text { Each }\end{array}$} \\
\hline $\begin{array}{c}\text { Reference } \\
\text { Temperature } \\
\left({ }^{\circ} \mathrm{C}\right)\end{array}$ & $\begin{array}{c}\text { Transmitter } \\
\text { Temperature } \\
\left({ }^{\circ} \mathrm{C}\right)\end{array}$ & $\begin{array}{l}\text { Transmitter } \\
\text { current }(\mathrm{mA})\end{array}$ & $\begin{array}{l}\text { Transmitter } \\
\text { Range (\%) }\end{array}$ & $\begin{array}{c}\text { Reference } \\
\text { Temperature } \\
\left({ }^{\circ} \mathrm{C}\right)\end{array}$ & $\begin{array}{c}\text { Transmitter } \\
\text { Temperature } \\
\left({ }^{\circ} \mathrm{C}\right)\end{array}$ & $\begin{array}{l}\text { Transmitter } \\
\text { current }(\mathrm{mA})\end{array}$ & $\begin{array}{l}\text { Transmitter } \\
\text { Range (\%) }\end{array}$ & \\
\hline 12.5 & 13.03 & 8.17 & 26.07 & 50 & 49.8 & 19.93 & 99.57 & 0 \\
\hline 25 & 18.02 & 9.93 & 37.58 & 37.4 & 43.63 & 17.91 & 86.46 & 2 \\
\hline 25 & 22.37 & 11.2 & 45.11 & 37.5 & 40.11 & 16.8 & 79.81 & 4 \\
\hline 25 & 24.01 & 11.7 & 48.18 & 37.5 & 38.41 & 16.27 & 76.61 & 6 \\
\hline 25 & 24.71 & 11.91 & 49.48 & 37.5 & 37.83 & 16.1 & 75.61 & 8 \\
\hline 25 & 24.93 & 11.98 & 49.88 & 37.5 & 37.55 & 16.01 & 74.83 & 10 \\
\hline 25 & 24.98 & 11.99 & 49.98 & 23.7 & 30.26 & 13.59 & 59.26 & 12 \\
\hline 25 & 25.02 & 12.01 & 50.05 & 23.7 & 25.84 & 12.25 & 51.43 & 14 \\
\hline 37.5 & 33.7 & 14.08 & 68.21 & 23.8 & 24.51 & 11.84 & 48.96 & 16 \\
\hline 37.5 & 36.28 & 15.62 & 72.72 & 23.8 & 24.21 & 11.74 & 48.38 & 18 \\
\hline 37.5 & 37.09 & 15.88 & 74.25 & 23.8 & 24.1 & 11.71 & 48.21 & 20 \\
\hline 37.5 & 37.36 & 15.96 & 74.74 & 13.2 & 21.36 & 10.75 & 42.11 & 22 \\
\hline 50 & 42.21 & 17.96 & 87.6 & 12.4 & 14.9 & 8.75 & 29.53 & 24 \\
\hline 50 & 47.12 & 19.1 & 94.67 & 12.5 & 13.32 & 8.26 & 26.6 & 26 \\
\hline 50 & 48.97 & 19.68 & 97.04 & 12.5 & 13.15 & 8.21 & 26.27 & 28 \\
\hline 50 & 49.52 & 19.85 & 99.07 & 12.5 & 13.11 & 8.19 & 26.19 & 30 \\
\hline 50 & 49.7 & 19.91 & 99.42 & 12.5 & 13.06 & 8.18 & 26.13 & 32 \\
\hline 50 & 49.76 & 19.92 & 99.52 & 12.5 & 13.03 & 8.17 & 26.09 & 34 \\
\hline 50 & 49.78 & 19.93 & 99.57 & 12.5 & 13.03 & 8.17 & 26.05 & 36 \\
\hline 50 & 49.8 & 19.93 & 99.57 & 12.5 & 13.01 & 8.17 & 26.03 & 38 \\
\hline
\end{tabular}

Table 4: Experimental results of a Rosemount 3144P intelligent temperature transmitter

\begin{tabular}{|c|c|c|c|c|c|c|}
\hline \multicolumn{4}{|c|}{ RISE } & \multicolumn{3}{|c|}{ FALL } \\
\hline $\begin{array}{c}\text { Reference } \\
\text { Temperature }\left({ }^{\circ} \mathrm{C}\right)\end{array}$ & $\begin{array}{c}\text { Transmitter } \\
\text { Temperature }\left({ }^{\circ} \mathrm{C}\right)\end{array}$ & $\begin{array}{c}\text { Percentage } \\
\text { Error (\%) }\end{array}$ & $\begin{array}{c}\text { Reference } \\
\text { Temperature }\left({ }^{\circ} \mathrm{C}\right)\end{array}$ & $\begin{array}{c}\text { Transmitter } \\
\text { Temperature }\left({ }^{\circ} \mathrm{C}\right)\end{array}$ & $\begin{array}{l}\text { Percentage } \\
\text { Error (\%) }\end{array}$ & Time (minutes) \\
\hline 12.5 & 13.03 & -1.06 & 50 & 49.8 & 0.4 & 0 \\
\hline 25 & 18.02 & 13.96 & 37.4 & 43.63 & -12.46 & 2 \\
\hline 25 & 22.37 & 5.26 & 37.5 & 40.11 & -5.22 & 4 \\
\hline 25 & 24.01 & 1.98 & 37.5 & 38.41 & -1.82 & 6 \\
\hline 25 & 24.71 & 0.58 & 37.5 & 37.83 & -0.66 & 8 \\
\hline 25 & 24.93 & 0.14 & 37.5 & 37.55 & -0.1 & 10 \\
\hline 25 & 24.98 & 0.04 & 23.7 & 30.26 & -13.12 & 12 \\
\hline 25 & 25.02 & -0.04 & 23.7 & 25.84 & -4.28 & 14 \\
\hline 37.5 & 33.7 & 7.6 & 23.8 & 24.51 & -1.42 & 16 \\
\hline 37.5 & 36.28 & 2.44 & 23.8 & 24.21 & -0.82 & 18 \\
\hline 37.5 & 37.09 & 0.82 & 23.8 & 24.1 & -0.6 & 20 \\
\hline 37.5 & 37.36 & 0.28 & 13.2 & 21.36 & -16.32 & 22 \\
\hline 50 & 42.21 & 15.58 & 12.4 & 14.9 & -0.5 & 24 \\
\hline 50 & 47.12 & 5.76 & 12.5 & 13.32 & -1.64 & 26 \\
\hline 50 & 48.97 & 2.06 & 12.5 & 13.15 & -1.3 & 28 \\
\hline 50 & 49.52 & 0.96 & 12.5 & 13.11 & -1.22 & 30 \\
\hline 50 & 49.7 & 0.6 & 12.5 & 13.06 & -1.12 & 32 \\
\hline
\end{tabular}




\begin{tabular}{|c|c|c|c|c|c|c|}
\hline \multicolumn{5}{|c|}{ RISE } & \multicolumn{3}{c|}{ FALL } \\
\hline $\begin{array}{c}\text { Reference } \\
\text { Temperature }\left({ }^{\circ} \mathrm{C}\right)\end{array}$ & $\begin{array}{c}\text { Transmitter } \\
\text { Temperature }\left({ }^{\circ} \mathrm{C}\right)\end{array}$ & $\begin{array}{c}\text { Percentage } \\
\text { Error }(\%)\end{array}$ & $\begin{array}{c}\text { Reference } \\
\text { Temperature }\left({ }^{\circ} \mathrm{C}\right)\end{array}$ & $\begin{array}{c}\text { Transmitter } \\
\text { Temperature }\left({ }^{\circ} \mathrm{C}\right)\end{array}$ & $\begin{array}{c}\text { Percentage } \\
\text { Error }(\%)\end{array}$ & Time (minutes) \\
\hline 50 & 49.76 & 0.48 & 12.5 & 13.03 & -1.06 & 34 \\
\hline 50 & 49.78 & 0.44 & 12.5 & 13.03 & -1.06 & 36 \\
\hline 50 & 49.8 & 0.4 & 12.5 & 13.01 & -1.02 & 38 \\
\hline
\end{tabular}

Table 5: Experimental results showing temperature resistance relationship

\begin{tabular}{|c|c|c|c|c|c|c|c|}
\hline \multicolumn{2}{|c|}{ RISE } & \multicolumn{3}{|c|}{ FALL } \\
\hline $\begin{array}{c}\text { Transmitter } \\
\text { Temperature } \\
\left({ }^{\circ} \mathrm{C}\right)\end{array}$ & $\begin{array}{c}\text { Transmitter } \\
\text { current }(\mathrm{mA})\end{array}$ & $\begin{array}{c}\text { Transmitter } \\
\text { RTD } \\
\text { Resistance } \\
(\mathrm{K} \Omega)\end{array}$ & $\begin{array}{c}\text { Transmitter } \\
\text { RTD } \\
\text { Temperature } \\
\text { Coefficient } \\
\left(\Omega /{ }^{\circ} \mathrm{C}\right)\end{array}$ & $\begin{array}{c}\text { Transmitter } \\
\text { Temperature } \\
\left({ }^{\circ} \mathrm{C}\right)\end{array}$ & $\begin{array}{c}\text { Transmitter } \\
\text { current }(\mathrm{mA})\end{array}$ & $\begin{array}{c}\text { Transmitter } \\
\text { RTD } \\
\text { Resistance } \\
(\mathrm{K} \Omega)\end{array}$ & $\begin{array}{c}\text { Transmitter } \\
\text { RTD } \\
\text { Temperature } \\
\text { Coefficient } \\
\left(\Omega /{ }^{\circ} \mathrm{C}\right)\end{array}$ \\
\hline 13.03 & 8.17 & 29.38 & 225.47 & 49.8 & 19.93 & 12.04 & 24.18 \\
\hline 18.02 & 9.93 & 24.17 & 134.13 & 43.63 & 17.91 & 13.40 & 30.71 \\
\hline 22.37 & 11.2 & 21.43 & 95.80 & 40.11 & 16.8 & 14.29 & 35.63 \\
\hline 24.01 & 11.7 & 20.51 & 85.42 & 38.41 & 16.27 & 14.75 & 38.40 \\
\hline 24.71 & 11.91 & 20.15 & 81.55 & 37.83 & 16.1 & 14.91 & 39.41 \\
\hline 24.93 & 11.98 & 20.03 & 80.34 & 37.55 & 16.01 & 14.99 & 39.92 \\
\hline 24.98 & 11.99 & 20.02 & 80.14 & 30.26 & 13.59 & 17.66 & 58.36 \\
\hline 25.02 & 12.01 & 19.98 & 79.86 & 25.84 & 12.25 & 19.59 & 75.81 \\
\hline 33.7 & 14.08 & 17.05 & 50.59 & 24.51 & 11.84 & 20.27 & 82.70 \\
\hline 36.28 & 15.62 & 15.36 & 42.34 & 24.21 & 11.74 & 20.44 & 84.43 \\
\hline 37.09 & 15.88 & 15.11 & 40.74 & 24.1 & 11.71 & 20.50 & 85.06 \\
\hline 37.36 & 15.96 & 15.04 & 40.26 & 21.36 & 10.75 & 22.33 & 104.54 \\
\hline 42.21 & 17.96 & 13.36 & 31.65 & 14.9 & 8.75 & 27.43 & 184.09 \\
\hline 47.12 & 19.1 & 12.57 & 26.68 & 13.32 & 8.26 & 29.06 & 218.17 \\
\hline 48.97 & 19.68 & 12.20 & 24.91 & 13.15 & 8.21 & 29.23 & 222.28 \\
\hline 49.52 & 19.85 & 12.09 & 24.41 & 13.11 & 8.19 & 29.30 & 223.49 \\
\hline 49.7 & 19.91 & 12.05 & 24.25 & 13.06 & 8.18 & 29.34 & 224.66 \\
\hline 49.76 & 19.92 & 12.05 & 24.22 & 13.03 & 8.17 & 29.38 & 225.48 \\
\hline 49.78 & 19.93 & 12.04 & 24.19 & 13.03 & 8.17 & 29.38 & 225.48 \\
\hline 49.8 & 19.93 & 12.04 & 24.17 & 13.01 & 8.17 & 29.38 & 225.83 \\
\hline
\end{tabular}

From the results shown in Table 5, it is seen that the temperature varies directly as the resistance of the temperature sensing device. This ultimately supports the view of IDC technologies [6] that electrical resistance increases with increased temperature.

To analyze the sensitivity of the RTD, we examine the magnitude of the temperature coefficient, which indicates how much the resistance changes with increase in temperature.

Temperature coefficient $=\frac{\text { Resistance }}{\text { temperature }}$

The temperature coefficient is seen from Table 5 to reach values of $225 \Omega /{ }^{\circ} \mathrm{C}$ indicating that the sensitivity of the RTD is high. It is also seen from Table 5 that sensitivity increases with decreased temperature.

\section{CONCLUSION}

From the analysis of the response of the Rosemount intelligent temperature transmitter to externally applied temperature, it is seen that the resistance of the platinum RTD varies directly with applied temperature as supported in literature. Also, the platinum RTD shows low response times with significantly high error margin for different simulated temperature. Error margins show acceptable values at $0.04 \%,-0.04 \%$ and $-0.1 \%$ respectively. This necessitates the need to implement manufacturer's recommended connection 
of resistor of value ranging from $250 \Omega$ to $1100 \Omega$ between current loop and transmitter in order to improve transmitter accuracy. The results also show that as RTD temperature increases, the temperature coefficient decreases.

The authors are presently exploring the possibility of exploiting the functionalities of the TeloB sensor node in capturing important physical parameters in a pipeline oil terrain based on its potential in ensuring improved commissioning time, improved reliability and troubleshooting and the capability of collaborative working. In future, the authors hope to compare experimental parameters of platinum PT 100 RTD with Sensirion SHT11 with a view of advising experts on most suitable device selection.

\section{REFERENCES}

[1] T.R. Kuphaldt. (2008) Lessons in industrial instrumentation. USA: Creative Commons attributions. pp.131-255.

[2] W. Boyes. (ed) (2010) Instrumentation reference book. $4^{\text {th }}$ edn. Burlington: Elsevier Inc. pp.1-22.

[3] I.F. Akyildiz, W. Su and Y. Sankarasubramaniam and E. Cayirci (2002) 'Wireless Sensor Networks: A Survey', Computer Networks,38, pp.393-422.

[4] C.Y. Chong and S. Kumar (2003) 'Sensor networks: evolution, opportunities and challenges', Proceedings of the IEEE, 91(8), pp. 1247-1256

[5] J.I. Bangash, A.H. Abdullah, M.H. Anisi and A.W. Khan (2014) 'A survey on Routing Protocols in wireless body sensor networks', Sensors, 14 (1), pp. 13221357.
[6] Rosemount Inc (1999) Rosemount model 3144 and 3244MV smart temperature transmitters. [Online]. Available at: http://www.rosemount.com (Accessed: 10th July 2014).

[7] Wireless HART Communication Protocol. http://www.hartcomm2.org/index.html

[8] IDC technologies (2012) Industrial automation. bookboon.com [Online]. Available at: http://www.bookboon.com/en/industrialautomation-ebook (Accessed: 20th August 2014). pp. 55-56.

[9] S.Y. Norf. (ed) (2009) Springer handbook of automation. Berlin Heidelberg: Springer.

[10] L. Gavrilovska, S. Krco, V. Milutinivic, I. Stojmenovic, and R. Trobec. (ed.s) (2011) Application and multidisciplinary aspects of wireless sensor networks. concepts, integration and case studies. London: Springer. pp. 13-33.

[11] P.Ripka and A.Tipek. (2007) Modern sensors handbooks. UK: ISTE Ltd.

[12] Ziehl Industrial Electronic (2014) Pt 100temperature sensors: type TF 101, D-74523. [Online]. Available at: http://www.ziehl.de (Accessed: 12th August 2014).

[13] I.F. Akyildiz, and M.C. Vuran. (2010) Wireless sensor networks. Chichester: John Wiley and Sons Ltd. pp. 1517.

[14] Advantic (2010) CM5000 datasheet. [Online]. Available at: http://www.advanticsys.com (Accessed: 20th September 2014).

[15] Sensirion (2011) Datasheet SHT1x humidity and temperature sensor IC. [Online]. Available at: http://www.sensirion.com (Accessed: 12th August 2014). 